\title{
"Touchscreen Assessment Tool" (TATOO), an Assessment Tool Based on the Expanded Conceptual Model of Frailty
}

\author{
Alexandra Danial-Saad ${ }^{1,2}$, Lorenzo Chiari $^{3}$, Yael Benvenisti ${ }^{4(\bowtie)}$, \\ Shlomi Laufer ${ }^{5}$, and Michal Elboim-Gabyzon ${ }^{6}$ \\ 1 Occupational Therapy Department, \\ Faculty of Social Welfare and Health Sciences, University of Haifa, Haifa, Israel \\ ${ }_{2}$ The Arab Academic College for Education in Israel, Haifa, Israel \\ 3 Department of Electrical, Electronic, and Information Engineering, \\ University of Bologna, Bologna, Italy \\ ${ }^{4}$ Mediterranean Towers Ventures, Ganei Tikva, Israel \\ yaelbenvenisti@gmail.com \\ ${ }^{5}$ Faculty of Industrial Engineering and Management, The Technion, \\ Haifa, Israel \\ ${ }^{6}$ Physical Therapy Department, Faculty of Social Welfare and Health Sciences, \\ University of Haifa, Haifa, Israel
}

\begin{abstract}
Frailty is a common clinical syndrome in older adults; it carries an increased risk of negative health events and outcomes including falls, incident disability, hospitalization, and mortality. Therefore, it is critical to identify highrisk subsets of the elderly population and explore new arenas for frailty prevention and treatment. This chapter will provide an overview of the current state of assessment models for frailty syndrome in the elderly and will describe a new assessment tool based on mobile technology, which takes account and advantage of the ways in which elderly people interact with a touchscreen. While healthcare providers and researchers in the field of aging have long been aware of the changing characteristics and needs of older people living in the community, there has not been any marked change in frailty syndrome assessment models until now. In the twenty-first century world with its technological advancements, the elderly require new, special physical skills combining perceptual, motor, and cognitive abilities for their functional daily activities and for maintaining their independence and quality of life.

We believe a conceptual model of frailty can be expanded to incorporate new aspects related to the usage of technology by the elderly, better covering the complexity and multidimensionality of modern life. In addition, in our vision, that the expanded conceptual model can be operationalized and translated into an assessment tool.

In the last part of this chapter, we will present the "Touchscreen Assessment Tool" (TATOO), an assessment tool based on this expanded conceptual model. This novel tool assesses elderly people's frailty and functioning using a touchscreen, a representative technology required for several activities involved
\end{abstract}


in daily functioning in the modern world. Most importantly, we present the TATOO prototype, which we plan to develop in the future as a continuous monitoring instrument for activities performed in daily life, combined with advanced sensor-based measuring and big data analytics algorithms.

Keywords: Frailty syndrome $\cdot$ Elderly $\cdot$ Touchscreen Assessment Tool

\section{Background}

Frailty syndrome is a common syndrome in the elderly, with a multi-factor etiology [1]. Frailty syndrome consists of the basic characteristic of increased susceptibility to biological, physiological, and mental stressors. Impairments in multiple organ systems (such as the musculoskeletal system, the cardiovascular system, and the hematological system) cause depletion in physiological and mental reserves and decrease resistance, thereby impeding the recovery ability of the elderly as well as their ability to maintain physiological and psychosocial homeostasis [2]. Accordingly, as previous studies have pointed out, frailty is related to a higher prevalence of disability, falls, hospitalization, and mortality in community-dwelling older people. It is a major predictor of clinical outcomes and prognosis following any extrinsic stress, such as medical procedure, acute illness, proximal hip fracture, or hospitalization [3-5].

The understanding of frailty has evolved over the years from a basic description of dependence on others to a more dynamic model that encompasses biomedical and psychosocial aspects. It depicts a complex interplay among a person's characteristics, such as age, gender, lifestyle, socioeconomic background, morbidities, and affective, cognitive, or sensory impairments. Currently, as there is no firm agreement among experts on the definition of frailty, it can be considered an amorphous concept, a situation that impedes our ability to identify it in the elderly and assess their condition.

Frailty, comorbidity, and disability are all common in the elderly, with overlaps and reciprocal interactions making it sometimes difficult to distinguish among them [6, 7]. Disability, defined as "difficulty or dependency in performing activities that are essential to independent living" [6], often worsens a person's frailty, but not all elderly with disabilities are frail and not all the frail elderly have disabilities [8].

A major requirement at present is to move from theoretical discussion on definitions of frailty to outlining practical and operational definitions that enable actual screening, assessment, and treatment of frailty, as well as differentiating frailty-related and unrelated conditions. For example, one major manifestation of frailty is functional decline, but functional decline in the elderly is part of the normal aging process and is not always an indicator of frailty.

Frailty is dynamic in nature and can be viewed as a process parallel to the process of aging; and age increases the risk of frailty [9]. The elderly population is heterogeneous in terms of chronological age and biological, psychological, and social factors, and hence, they cannot be considered a single entity. Thus, researchers often group older adults by chronological age, for example, considering adults ranging in the age group 60-75 years as a younger-old group, and individuals over 75 as an older-old 
group [10]. The challenge is then to find a point in time when a given elderly patient moves from only aging to becoming frail as well.

Despite being progressive and chronic, frailty syndrome is also reversible in the pre-frailty stage and in the early stages of frailty; accordingly, screening of the elderly population is required for early detection of elderly who are frail or at risk of becoming frail. Continuous monitoring, preventive interventions, and treatment programs should be made routine in healthcare for the ever-growing elderly population all over the world [11, 12]. In contrast to the early stages, advanced frailty syndrome is irreversible, but its rate of progression can still be reduced to some extent, with the possibility of reducing and preventing complications and improving quality of life [13].

A crucial need in this regard is to operationalize the concept of frailty by developing validated, reliable, feasible assessment tools to capture all the clinical aspects of geriatric frailty. It should be emphasized that diagnosing an older person as frail has implications at several levels, ranging from the individual to health policy and systems.

The complexity of frailty and the difficulty in distinguishing between natural aging and pathological conditions have led to the development of models and instruments that attempt to comprehensively cover all the aspects of the condition [5, 14-16]. Numerous diagnostic tools for frailty have been developed in recent years. A systematic review by Buta et al. [17] identified 67 frailty instruments, of which only 9 were highly cited. The instruments most used are the physical frailty phenotype, followed by the frailty index (also called the "deficit accumulation index") [17].

The physical frailty phenotype is the single most widely used instrument for assessing frailty [17], and is based on the frailty phenotype model developed by Fried et al. [5]. It stipulates a cluster of consensus elements of clinical presentation of frailty: age-related changes in body mass, muscle strength, endurance, pattern of walking, and level of physical activity $[5,14,18]$. The crucial points involved in frailty versus normal age-related change in this model are the existence of multiple impaired elements and the degree of change. The physical phenotype of frailty was operationalized by Fried et al. and validated by the Cardiovascular Health Study [5]. As per the study, frailty is characterized by the presence of three or more of the following five components: (1) unintentional weight loss (more than $4.55 \mathrm{~kg}$ in a year), (2) muscle weakness (grip strength in the lowest $20 \%$ at baseline, adjusted for gender and body mass index), (3) poor endurance and energy (evaluated by self-report of exhaustion), (4) slow walking speed (time to walk $15 \mathrm{ft}$, adjusting for gender and standing height), and (5) low physical activity level (measured by kilocalories expended per week). Scores on these parameters are categorized into three levels of frailty: (1) robustness (none of the criteria), (2) pre-frailty (one or two criteria), and (3) frailty (three or more criteria) [5].

Another common tool is the frailty index, developed by Rockwood et al. [19], which comprises 70 items on the presence and severity of diseases, ability to perform activities of daily living (ADLs; e.g., getting dressed, bathing, mobility), and physical and neurological signs from clinical examinations. The frailty index focuses on objective measures of the amount of accumulated deficits/functional losses, reflecting the degree of exhaustion of reserves [16]. 
Cesari et al. [16] compare the physical phenotype of frailty and the frailty index and find that the two tools provide "distinct and complementary clinical information about the risk profile of an older person" [16]. The authors suggest that the purposes of the tools are different and that their respective usage should be determined by the timing of the assessment, with physical frailty phenotype tool used as a screening tool for quick identification of frailty as a gross category and the frailty index as part of a comprehensive geriatric assessment to serve as reference data for following the elderly over time, making subsequent assessments, and examining the efficiency of targeted interventions [16].

The existence of numerous measurement tools indicates a lack of agreement on the conceptual framework and components of frailty, appropriate assessment items, purpose of use (risk assessment, etiology, research methodologies design), clinical practicality, and differentiating between disability and frailty [17, 20]. However, efforts to establish consensus are ongoing and the seeds of agreement can be seen [17, 21, 22].

\section{Operational Definitions of Frailty}

It is recognized that operational definitions of frailty should be: (1) multi-dimensional, based on the connections between physical, psychological, and social domains, and not constrained to physical function; (2) clearly distinct from disability; (3) cognizant of comorbidity (presence of $\geq 2$ diseases); (4) proven valid for predicting negative outcomes; (5) feasible; and (6) continuous and not categorical in terms of scoring.

Measurement of frailty should be based on continuous scores due to the nature of frailty, which is a progressive process. Raphael et al. [23] claimed that the degree of frailty on that continuum is a result of the interaction between personal factors and environmental factors. They described both types of factors in detail: "'personal factors' include the immediate cognitive (e.g., memory loss), physical (e.g., reduced mobility), psychological (e.g., depression, lack of self-efficacy), and spiritual factors (e.g., loss of hope or meaning), while 'environmental factors' include financial, social (e.g., availability of friends or family), living situation (e.g., many steps, danger in one's neighborhood), and legal (e.g., not being able to drive because of legislation) factors."

We believe that using technology can be crucial in integrating personal and environmental factors. The novel assessment tool described here is based on "personenvironment interactions," and it tries to overcome the insufficient emphasis on the environmental aspect reflected in previous tools by leveraging the common use of technology in ADLs in the modern world. Technologies such as touchscreens, voice recognition systems, artificial intelligence, etc., are becoming increasingly prevalent in daily life among the general population and considerably more among older adults [24]. 
The traditional methods for assessing frailty syndrome in the elderly, however, do not use these emerging technologies, which facilitate continuous, passive, objective assessments. A tool reflecting their technological affordances can considerably improve accuracy, including by reflecting the context (a person's natural environment).

Evidence for this approach has been shown in recent years in healthcare in general, with medical tests that no longer rely solely on subjective evaluations but also employ objective evaluations using advanced algorithms [25, 26].

Moreover, since the percentage of elderly people in the world is increasing along with their life expectancy, clinicians cannot rely on individual face-to-face meetings to evaluate and monitor each person over time because of both the economic costs involved in these tests and the lack of manpower [27]. Hence, to diagnose and monitor over time the frailty of an older person and allow him/her to receive treatment/intervention to improve his/her condition, or prevent deterioration, we as a society must develop new, effective, inexpensive technology-based assessment tools. Figure 1 below illustrates the components that should be included in the modern assessment of frailty.

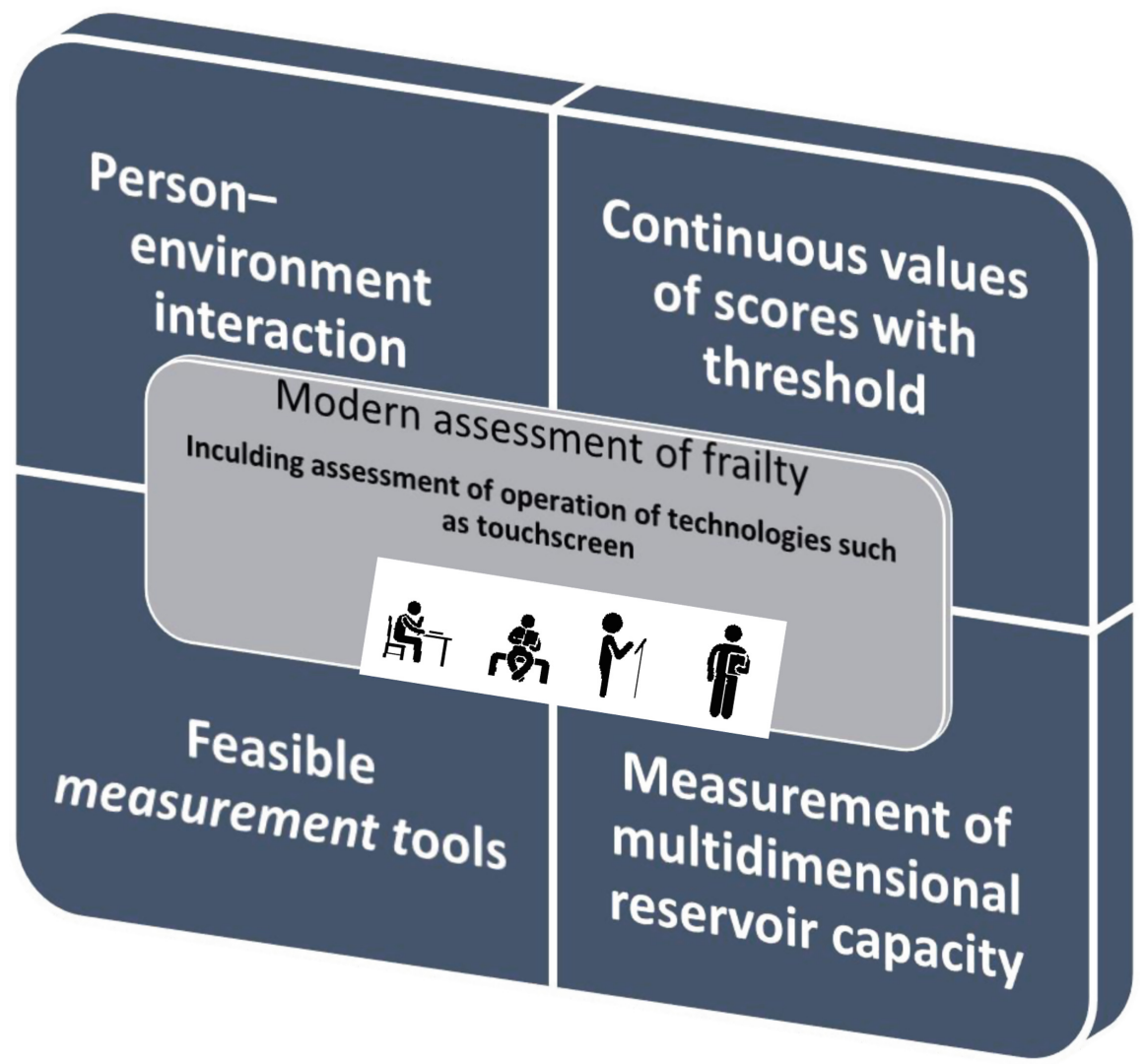

Fig. 1. Mandatory components of frailty assessment 


\section{A Touchscreen Tool for Assessment Based on the Expanded Conceptual Model of Frailty}

We believe that the conceptual framework of frailty is incomplete and does not cover all the aspects of the real life of individuals in the modern world. The model should be extended to reflect the performance of the elderly engaged in daily activities in an environment in which technology is embedded in each one of the three components of the human function (physical, psychological, and social). In today's highly technologyoriented world, elderly individuals are obliged to interact safely and proficiently not only with technological applications designed for their benefit, but also with multiple technological devices developed for the general (and often young) population.

The use of a touchscreen is an excellent example of a technological application that has become prevalent in all aspects of modern life. Due to the convenience, design flexibility, and ease involved in manipulating touch interfaces [28], this technology is frequently used in public settings and is one of the most dominant ways by which the elderly interact with modern computing devices [28]. Touchscreens are now used by the elderly to perform multiple daily functional activities in pursuing their social, personal and occupational activities, ranging from buying a ticket for public transportation, ordering food in a restaurant, withdrawing money from an ATM, or accessing health services and health information. Using a touchscreen is also related to the social and leisure domains of human functions as it is used for communicating via smartphone and for personal interactions via social media (such as Facebook); hence, it may reflect the degree of social inclusion and social support the elderly have in coping with loneliness issues.

Our idea is consistent with Nourhashémi et al. [22] who asserted that frailty in elderly persons should be assessed in the context of a combination of not only biological, physiological, and social changes, but also environmental changes. The modern world is characterized by such rapid changes in the several aspects of daily life, with a growing pervasiveness of technological components. These technologies should enhance the elderly's independence in life's roles, make their leisure activities more meaningful, and enhance their self-esteem.

Following the modification of the frailty model, an operational definition and an assessment tool are required to measure the ability of the elderly to operate technology, in this case, the touchscreen (see Fig. 1).

The transfer from normal aging into dynamic frailty status is a continuous process that can be investigated through changes in how operating a touchscreen, as an example of a pervasive technology of daily use, changes over time. We assume that operating the touchscreen may be a sensitive way of detecting early signs of frailty and, accordingly, that it can be used as a screening tool for quick identification of pre-frailty as a gross category with the potential of further development once integrated into a comprehensive geriatric assessment to serve as reference data for following the elderly over time and for examining the efficacy of the targeted interventions. 


\section{The TATOO and Its Importance}

In recent years, research and clinical studies have focused on finding ways and criteria to assess the status of the elderly people, in order to identify situations in which support is needed to enable their continued independent active aging in the community. An increasing part of daily functioning involves the use of touchscreens to perform tasks in various areas of life, such as communication, administrative tasks, healthcare, etc. The specific physical, perceptual, and cognitive skills required to use touchscreens may be affected by a multisystem decline in body functions and capabilities that occur during aging, such as decreased reaction speed, worsening eye-hand coordination, decreased muscle strength, and sensory decline [1]. Thus, differences in the ability to successfully operate a touchscreen are expected between young and old adults. These differences may be intensified in frail older adults and in older adults with age-related pathological conditions, such as arthritis, stroke, or Parkinson's disease. While most age-related physiological changes (e.g., cardiovascular changes) affect human performance, the body systems most relevant to the operation of a touchscreen are the sensory-perceptual system, the psychomotor system, and cognition.

Therefore, it is essential to identify evaluation tools that can measure the actual interaction between an elderly person and a touchscreen, as a representation of many technological interfaces that require similar skills. Furthermore, a well-fitted technology may contribute to the elderly by engage them in basic and instrumental activities of daily living, enhance their independence, provide meaningful leisure activities, and help them acquire more self-esteem and become productive members of mainstream society. The process of achieving an optimal match between technology and the elderly should involve an assessment of their needs, emotional state, preferences, and acceptance.

Among technology professionals who play a central role in prescription, provision, and adaptation of devices to meet the elderly's needs, there is a lack of dependable assessment tools to determine elderly behavior and needs while using touchscreen devices. Daniel-Saad and Chiari [24] developed a software application called TATOO to evaluate performance components of the use of touchscreens in a comprehensive and objective manner. The tool was developed by gaining a consensus regarding the user skills required to operate various touchscreen devices. A six-step procedure was used to collect and validate the required skills by a multidisciplinary team of 52 experts. TATOO consists of six tasks, each of which provides information on a functional component required when using a touchscreen, such as tap, swipe, pinch, drag, and so on. Performance of each task is summarized by a numeric and graphic report of the following parameters:

1. Timing: includes Start Time, Reaction Time, Test Duration, Total Touch Time (the time the finger is on the screen while tapping and dragging), and Total Flight Time (the time the finger is up in the air between drags).

2. Movement range and pressure measurement: the report shows a graphical "taps map and path drawing" representing the location of the taps, the path the client has used to drag, and the pressure applied while executing each drag.

3. Mode of operation: the number of fingers used to operate for each skill. 
4. Accuracy: the report shows numerical and graphical information that presents trials that were completed successfully or not completed. The information includes the location of the first tap on the screen, Total Touch Outside the Target, Total Drag Attempts, and Drag Completed Successfully or Not Completed.

The TATOO was used primarily to assess touchscreen performance by children. Currently, we are in the process of adjusting the system to assess the performance of the elderly population, particularly focusing on the detection of early stages of frailty. The system can be used for routine and periodic evaluation of the elderly by healthcare personnel. Health professionals such as physicians, occupational therapists, physical therapists, or nurses can use TATOO because of its easy method of operation, which is not time consuming to learn or implement. Information supplied by TATOO provides objective data that enable clinical decisions (such as prescribing treatment) by multidisciplinary healthcare professionals. Integrating the system to the healthcare services will enable early assessment of change or regression in the visual-perceptual motor function of the elderly to enable early intervention and prevention of reversible deterioration or complication.

The current study was approved by the Helsinki Committee of the University of Haifa. The sample will be composed of 300 elderly people divided into three age groups as follows: 1. Younger adults (40-65 years); 2. Young-old adults (65 to 75 years) and 3. Older-old adults (over 75 years). The inclusion criteria are: ability to understand simple commands; living independently in the community or in independent living facilities; and ability to walk independently with or without walking aid. Exclusion criteria are: neurological diseases and severe orthopedic and cardiopulmonary conditions that may affect performance of daily activities; pain, impairments, or medical conditions that may prohibit performance of required activities; and serious uncorrected vision or hearing impairment. Younger adults will be recruited from the institutes' campus; young-old and older-old adults will be recruited from community centers for the elderly. All participants will provide written consent following a detailed explanation of the study.

The study will include three stages: 1. performance in a battery of clinical assessments to characterize the participants' physical and cognitive status and level of frailty; 2. capturing of data by the TATOO; 3 . Data analysis.

Stage 1: Characterization of the participants. The following information will be collected from all the eligible and consenting participants: (a) socio-demographic data (such as age, gender, marital status, years of education, use of the Internet, frequency of use of touch screens, level of income); (b) medical history. For participants from the two elderly groups, additional evaluation will be done to characterize them in term of (a) level of functioning in performing basic daily activities, measured by Index of Independence in Activities of Daily Living - Katz index; (b) fear of falling, using the Activities-specific Balance Confidence (ABC) Scale, (c) cognitive status, by the Montreal Cognitive Assessment (MoCA) questionnaire; (d) fine motor performance; and (e) level of frailty (measured by frailty phenotype instrument). It should be mentioned that the frailty phenotype instrument includes assessment of bilateral handgrip strength using a calibrated JAMAR hand dynamometer (Sammons Preston Rolyan, IL, USA) and walking speed measured by stopwatch. In addition, balance ability is 
measured by the "timed Up \& Go" (TUG) test (an index for dynamic balance function). Fine motor performance is measured by calibrated hydraulic pinch gauge (Ernest Bontage) and by the Functional Dexterity Test (FDT).

Stage 2: Capture of data by the TATOO system. Following the performing of the six TATOO tasks, which will last for approximately $10 \mathrm{~min}$, each participant will evaluate TATOO usability and user satisfaction by completing the System Usability Scale (SUS).

Stage 3: Data Analysis. We will determine the correlations between TATOO and performance measures as well as frailty level, and create a composite score which will include traditional measures and TATOO performance.

In a preliminary pilot study with nine older-old adults (mean age 80.4, SD 4.5) the TATOO's usability was rated as "very good" (average score of SUS 77.5) in terms of: effectiveness, efficiency and user satisfaction. The two healthcare professionals who conducted the trials reported that TATOO, from their point of view, was very user friendly in terms of short time assessment. Moreover, the interface was easy to learn and use, and the tool motivated the elderly to cooperate with the healthcare professionals.

Preliminary analysis demonstrates that young age, previous experience with a touch screen device, and high frequency of usage of touch screen positively correlate with better performance ability of the TATOO in term of timing and accuracy. Additionally, the amount of pressure on the screen is related to the accuracy requirements for task completion.

Conclusions regarding the correlation between TATOO scores and the degree of frailty of the elderly will be possible only after completing the current study with a large sample of participants ranging between younger and older-old adults.

Following is a presentation of the TATOO performance of a single female subject (initials HV), age 79 years. This subject is categorized as robust as defined by physical frailty phenotype, is independent in all activities of daily living, and presents with grip strength within the normal range per age. In contrast, however, HV presents with a low score (22/30) in the MOCA, indicating mild cognitive impairment. In addition, the fine dexterity measurement scores of both hands were very low, indicating low neuromotor function of the hand. TATOO performance was characterized by the use of one finger to tap the screen when the time duration of the task was short and the task was easy; when the time duration was longer, or the task was more complex, the pattern of tapping changed to using more than one finger.

HV's TATOO accuracy parameters were low. For example, she made 17 attempts to drag the five items of clothing, and in 14 of these attempts she was unable to place the item correctly. (see Fig. 2 for screen display). This finding was consistent with her low score on the Fine Dexterity Test (FDT; 61.3 seconds) performed by the dominant hand. 


\begin{tabular}{|c|c|}
\hline Start Time: & 16:02 \\
\hline Reaction Time [sec]: & 2.81 \\
\hline Test Duration [sec]: & 35 \\
\hline Total Fight Time [sec]: & 22 \\
\hline Total Touch Tome [sec]: & 17.5 \\
\hline Total Drag Attremps: & 14 \\
\hline Drag Completed Successfully: & 3 \\
\hline Drag Nor Completed: & 0 \\
\hline Toral Touch Outside the Target: & 17 \\
\hline Touch with Nultr-Fingers: & 7 \\
\hline
\end{tabular}

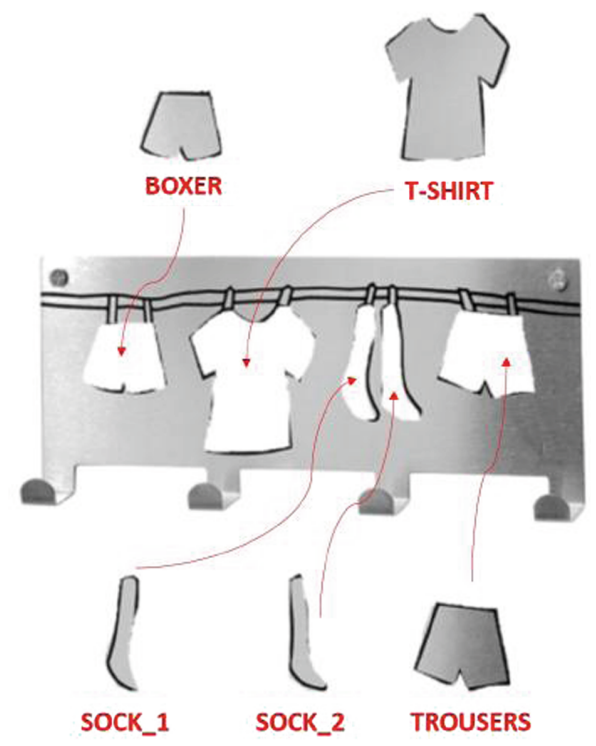

Fig. 2. Example of a screen display during a TATOO test, which includes the task and performance data of variables such as total touch time and touch time outside the target. (Color figure online)

\section{Practical Implications}

Frailty among the elderly leads to an increase in the use of health and welfare services and an increase in morbidity and mortality. Developing modern screening tools that are sensitive to early identification of frailty in the elderly and continuous monitoring is essential at the personal, national, and international levels. Knowledge of the course of development of frailty will enable identification of at-risk elderly and contribute to planning interventions and treatments to monitor and assist in situations of decreased physical and cognitive functioning. This will inevitably lead to better health outcomes, more disability-adjusted life years, and improved quality of life, as well as bringing economic savings [3].

The TATOO is a novel operational translation of an extended concept model of frailty of the elderly. It may become an important supplement to the toolbox available 
to clinical professionals treating the elderly. The current prototype provides quick, integrated information regarding the actual status of the elderly in relation to three crucial elements (fine motor, cognitive, and perceptual skills) reflecting performance and independence in basic and instrumental activities of daily living. Important point that should be emphasized is that TATOO has the potential to provide unique information that can be added to existing frailty indicators such as muscle weakness and slow walking speed. However, further studies should be performed to analyze the relative contribution and level of sensitivity and specificity of TATOO as a frailty screening and monitoring tool, comparing it to other frailty indicators.

\section{References}

1. Markle-Reid, M., Browne, G.: Conceptualizations of frailty in relation to older adults. J. Adv. Nurs. 44(1), 58-68 (2003)

2. Bergman, H., Ferrucci, L., Guralnik, J., et al.: Frailty: an emerging research and clinical paradigm-issues and controversies. J. Gerontol. A 62(7), 731-737 (2007)

3. Kim, D.H., Schneeweiss, S., Glynn, R.J., Lipsitz, L.A., Rockwood, K., Avorn, J.: Measuring frailty in medicare data: development and validation of a claims-based frailty index. J. Gerontol. A 73(7), 980-987 (2017)

4. Kojima, G.: Frailty as a predictor of disabilities among community-dwelling older people: a systematic review and meta-analysis. Disabil. Rehabil. 39(19), 1897-1908 (2017)

5. Fried, L.P., Tangen, C.M., Walston, J., et al.: Frailty in older adults: evidence for a phenotype. J. Gerontol. A 56(3), M146-M157 (2001)

6. Campbell, A.J., Buchner, D.M.: Unstable disability and the fluctuations of frailty. Age Ageing 26(4), 315-318 (1997)

7. Gobbens, R.J., Luijkx, K.G., Wijnen-Sponselee, M.T., Schols, J.M.: Toward a conceptual definition of frail community dwelling older people. Nurs. Outlook 58(2), 76-86 (2010)

8. Fried, L.P., Ferrucci, L., Darer, J., Williamson, J.D., Anderson, G.: Untangling the concepts of disability, frailty, and comorbidity: implications for improved targeting and care. J. Gerontol. A 59(3), M255-M263 (2004)

9. Robinson, T.N., Eiseman, B., Wallace, J.I., et al.: Redefining geriatric preoperative assessment using frailty, disability and co-morbidity. Ann. Surg. 250(3), 449-455 (2009)

10. Czaja, S.J., Rogers, W.A., Fisk, A.D., Charness, N., Sharit, J.: Designing for Older Adults: Principles and Creative Human Factors Approaches. CRC Press, London (2009)

11. Fried, L.P., Hadley, E.C., Walston, J.D., et al.: From bedside to bench: research agenda for frailty. Sci. Aging Knowl. Environ. 2005(31), 24 (2005)

12. Sacha, J., Sacha, M., Soboń, J., Borysiuk, Z., Feusette, P.: Is it time to begin a public campaign concerning frailty and pre-frailty? A review article. Front. Physiol. 8, 484 (2017)

13. Chen, X., Mao, G., Leng, S.: Frailty syndrome: an overview. Clin. Interven. Aging 9, 433 (2014)

14. Chin, A., Paw, M.J., Dekker, J.M., Feskens, E.J., Schouten, E.G., Kromhout, D.: How to select a frail elderly population? A comparison of three working definitions. J. Clin. Epidemiol. 52(11), 1015-1021 (1999)

15. Rockwood, K., Fox, R.A., Stolee, P., Robertson, D., Beattie, B.: Frailty in elderly people: an evolving concept. CMAJ 150(4), 489 (1994)

16. Cesari, M., Gambassi, G., Abellan van Kan, G., Vellas, B.: The frailty phenotype and the frailty index: different instruments for different purposes. Age Ageing 43(1), 10-12 (2013) 
17. Buta, B.J., Walston, J.D., Godino, J.G., et al.: Frailty assessment instruments: systematic characterization of the uses and contexts of highly-cited instruments. Ageing Res. Rev. 26, 53-61 (2016)

18. Winograd, C.H., Gerety, M.B., Chung, M., Goldstein, M.K., Dominguez Jr., F., Vallone, R.: Screening for frailty: criteria and predictors of outcomes. J. Am. Geriatr. Soc. 39(8), 778$784(1991)$

19. Rockwood, K., Song, X., MacKnight, C., et al.: A global clinical measure of fitness and frailty in elderly people. CMAJ 173(5), 489-495 (2005)

20. Cesari, M., Prince, M., Thiyagarajan, J.A., et al.: Frailty: an emerging public health priority. J. Am. Med. Dir. Assoc. 17(3), 188-192 (2016)

21. Rodriguez-Mañas, L., Fried, L.P.: Frailty in the clinical scenario. Lancet 385(9968), e7-e9 (2015)

22. Nourhashémi, F., Andrieu, S., Gillette-Guyonnet, S., Vellas, B., Albarède, J.L., Grandjean, H.: Instrumental activities of daily living as a potential marker of frailty: a study of 7364 community-dwelling elderly women (the EPIDOS study). J. Gerontol. A 56(7), M448M453 (2001)

23. Raphael, D., Cava, M., Brown, I., et al.: Frailty: a public health perspective. Can. J. Publ. Health 86(4), 224-227 (1995)

24. Danial-Saad, A., Chiari, L.: A multidisciplinary approach for developing an assessment tool for touch screen devices. Disabil. Rehabil. Assist. Technol. 13, 1-9 (2017)

25. Erickson, B.J., Korfiatis, P., Akkus, Z., Kline, T.L.: Machine learning for medical imaging. Radiographics 37(2), 505-515 (2017)

26. Lee, J.G., et al.: Deep learning in medical imaging: general overview. Korean J. Radiol. 18 (4), 570-584 (2017)

27. Sirven, N., Rapp, T.: The cost of frailty in France. Eur. J. Health Econ. 18(2), 243-253 (2017)

28. Caprani, N., O’Connor, N.E., Gurrin, C.: Touch screens for the older user. In: Auat Cheein, F. (ed.) Assistive Technologies. IntechOpen, London (2012)

Open Access This chapter is licensed under the terms of the Creative Commons Attribution 4.0 International License (http://creativecommons.org/licenses/by/4.0/), which permits use, sharing, adaptation, distribution and reproduction in any medium or format, as long as you give appropriate credit to the original author(s) and the source, provide a link to the Creative Commons licence and indicate if changes were made.

The images or other third party material in this chapter are included in the chapter's Creative Commons licence, unless indicated otherwise in a credit line to the material. If material is not included in the chapter's Creative Commons licence and your intended use is not permitted by statutory regulation or exceeds the permitted use, you will need to obtain permission directly from the copyright holder.

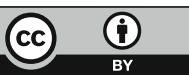

